\title{
Near-infrared multiwavelength imaging polarimetry of the low-mass proto-stellar object HL Tauri (Research Note)
}

\author{
K. Murakawa ${ }^{1}$, S. Oya ${ }^{2}$, T.-S. Pyo ${ }^{2}$, and M. Ishii ${ }^{2}$ \\ 1 Max-Planck-Institut für Radioastronomie, Auf dem Hügel 69, 53121 Bonn, Germany \\ e-mail: murakawa@mpifr-bonn.mpg.de \\ 2 Subaru Telescope, National Astronomical Observatory of Japan, 650 North A'ohoku place, Hilo, HI 96720, USA \\ Received 31 July 2008 / Accepted 15 October 2008
}

\section{ABSTRACT}

\begin{abstract}
We present the $J H K$-band high-resolution polarimetric images of the low-mass proto-stellar object HL Tau using the adaptive opticsequipped CIAO instrument on the Subaru telescope. Our polarization images show a butterfly-shaped polarization disk with an $\sim 0$ ' $9 \times$ 3". 0 extension. In the nebula, where polarization vectors are centro-symmetrically aligned, the polarization is as high as $P_{J} \sim 30 \%$, $P_{H} \sim 42 \%$, and $P_{K} \sim 55 \%$. On the other hand, low polarizations of $P<3 \%$ in the $J, H$, and $K$ bands and a low color excess ratio of $E_{J-H} / E_{H-K}=1.1$ compared to the standard cloud value of 1.75 are detected towards the central star. We estimated the upper limit of the grain sizes $a_{\max }$ to be $0.4 \mu \mathrm{m}$ in the nebula and $\gtrsim 0.7 \mu \mathrm{m}$ in the line of sight towards the central star. Our high-resolution polarimetric data, which spatially resolves the polarization disk, provides us with important information about grain growth in the region close to the central star.
\end{abstract}

Key words. polarization - ISM: dust, extinction

\section{Introduction}

HL Tau is an extensively studied, representative low mass young stellar object (YSO) which belongs to the L1551 Taurus dark cloud. The central star is deeply embedded in the circumstellar material (Stapelfeldt et al. 1995), and high velocity, wellcollimated jets with opening angles of $5^{\circ}-10^{\circ}$ were detected (e.g. Mundt \& Fried 1983; Ray et al. 1996; Pyo et al. 2006). The spectral energy distribution of this object shows a flat structure in the mid- to far-infrared, suggesting that this object is transiting from the proto-stellar to the T Tauri phase (Calvet et al. 1994). Also taking into account detections of evidence for an infalling motion (e.g. Hayashi et al. 1993) and surrounding obscuring material (Stapelfeldt et al. 1995) suggests that the cloud material is still accreting onto the disk (see also Adams et al. 1987; Kenyon \& Hartmann 1987; Bertout et al. 1988).

The disk is a key component of young stellar objects because the physical structure is characterized by the star-forming process, and it is a field where grain growth progresses. However, it is difficult to directly detect the images and investigate the physical properties because of small angular sizes; e.g. a 0. '7 radius for a typical T Tauri disk radius of 100 AU at a distance of 140 pc. High-resolution techniques such as adaptive optics (AO), speckle interferometry, and infrared interferometry achieve sub-0!' 1 angular resolutions which can, in theory, resolve typical T Tauri disks if objects are in nearby star-forming regions. It is also known that NIR imaging polarimetry is a powerful technique to derive dust properties and geometry structures via dust scattered light. For example, bipolar nebulae seen nearly edge-on show a centro-symmetric polarization vector pattern in the lobes on a large scale and a polarization disk in the equatorial plane (Kenyon et al. 1993; Whitney et al. 1997;
Perrin et al. 2004). These signatures are explained with a model consisting of a disk/torus and infalling envelope (Bastien \& Ménard 1988; Whitney \& Hartmann 1993; Fischer et al. 1996). Furthermore, our recent experiments have demonstrated that multiwavelength, high-resolution imaging polarimetry allows us to also derive multiple grain populations; e.g., large grains in the equatorial disk region and small grains in the surrounding nebula (Murakawa et al. 2008a,b,c). In this paper, we present $J H K$-band high-resolution polarimetric images of the low mass protostar HL Tau and discuss the polarization properties and the grain sizes in the disk and envelope.

\section{JHK-band imaging polarimetry}

\subsection{Observations}

We obtained the polarimetric images of HL Tau in the $J, H$, and $K$ bands using the AO-equipped near-infrared (NIR) camera CIAO on the $8 \mathrm{~m}$ Subaru telescope on December 27, 2006. The natural seeing was $\sim 0 . ' 5$ in the $R$ band during our observations. Since the target is bright enough at a wavelength for the wavefront sensing $\left(m_{R}=11.4\right)$, on-axis AO wavefront sensing was applied. To measure linear polarization, a turnable halfwave plate (HWP) and a fixed wire-grid polarizer were used, and we obtained four sets of images with HWP orientations of $0^{\circ}, 45^{\circ}, 22.5^{\circ}$, and $67.5^{\circ}$. At each band, four jitter positions were applied and the four sets of images above were obtained at each jitter position. The total integration times were $960 \mathrm{~s}$ for the $J$ band, $1280 \mathrm{~s}$ for the $H$ band, and $960 \mathrm{~s}$ for the $K$ band. We also observed NGC $1333 \mathrm{SVS} 13\left(m_{J}=11.67\right.$, $P_{J}=6.81 \pm 0.02 \%, m_{H}=9.65, P_{H}=5.02 \pm 0.03 \%, m_{K}=8.17$, 
and $P_{K}=3.41 \pm 0.04 \%$ ) for polarization calibration (Whittet et al. 1992).

We reduced the observed data as described in our previous papers (Murakawa et al. 2004, 2005). After subtraction of the dark frame and flat-fielding, we obtained images of the Stokes $I Q U$ parameters, the polarized intensity $(P I)$, the degree of linear polarization $(P)$, the polarization orientation $(\theta)$, as well as their error images. We found signal-to-noise ratios (SNs) of the Stokes $I$ images of $\mathrm{SN}_{J}=30-300, \mathrm{SN}_{H}=30-300$, and $\mathrm{SN}_{K}=20-200$ per pixel and the errors of linear polarization of $P_{J}<3 \%, P_{H}<3 \%$, and $P_{K}<5 \%$ per pixel within $\sim 2$ " from the central star. The accuracy of the polarization orientation per pixel is $<2.0^{\circ}$ in the lobes. The estimated PSF sizes are $\sim 0$ ' 2 in the $J$ band and $\sim 0$.' 1 in the $H$ and $K$ bands.

\subsection{Results}

Figure 1 shows the intensity (Stokes $I$ ), color, polarized intensity, and degree of linear polarization images with the polarization vector lines in the $J, H$, and $K$ bands. In the intensity images (top panels), we see an extended nebulosity with an arc-like feature in the north with a $\sim 5^{\prime \prime}$ extension and a C-shaped structure with an $\sim 11^{\prime \prime} 5$ extension near the central star. The central star is seen in the $H$ and $K$ bands, but is almost obscured in the $\mathrm{C}$-shaped feature in the $J$ band. These appearances are consistent with the previously reported images (e.g. Beckwith \& Birk 1995; Stapelfeldt et al. 1995; Close et al. 1997).

The second panels in Fig. 1 show the color index images of $J-H$ (left), $H-K$ (middle), and $(J-H) /(H-K)$ (right). We see some spots with low $J-H$ values $(\sim 0.7)$ near the central star, which approximately trace the C-shaped feature, and a high value of $7.6 \mathrm{mag}$ towards the central star in the $J-H$ image. We also find a different color pattern between the east and west sides of the nebula, which is split by a long arc-like feature extending from the north to the east, crossing near the central star. The east side has a $J-H$ of $\sim 4 \mathrm{mag}$, an $H-K$ of $\$ 2 \mathrm{mag}$, and a $(J-H) /(H-K)$ of 2-3.5. On the other hand, lower $J-H \sim 2 \mathrm{mag}$, higher $H-K \sim 3 \mathrm{mag}$, and lower $(J-H) /(H-K)$ of $0-1$ are detected on the western side. The $H-K$ color index pattern can be explained by a stronger extinction in the southwest lobe than in the northeastern lobe because the northeastern side of the HL Tau system is tilted towards the observer (Hayashi et al. 1993; Mundy et al. 1996). In contrast, the $J-H$ color index pattern does not agree with the simple extinction model above. One reason could be due to a complicated optical depth structure; i.e. the northeastern part is optically thin in the $H$ and $K$ bands, but it becomes optically thick in the $J$ band. The color information towards the central star indicates the reddening by dust. If we assume the intrinsic color of the central star to be $(J-H)_{\text {int }}=0.6$ and $(H-K)_{\text {int }}=0.15$, which is obtained from an estimated stellar temperature of $\sim 4000 \mathrm{~K}$ (e.g. Men'shchikov et al. 1999), the color excess values and their ratio are estimated to be $E_{J-H}=7.3, E_{H-K}=6.4$, and $E_{J-H} / E_{H-K}=1.1$. The color index ratio $E_{J-H} / E_{H-K}$ is significantly lower than an estimated average value of 1.75 for the Taurus dark cloud (Whittet et al. 2007), suggesting larger grain sizes close to the central star compared to the ones in the parent molecular cloud.

In the polarized intensity images (the third row), the northern arc, the C-shaped feature, and the southwestern lobe show high polarized intensity and the southwestern feature is split by the dark lane, which corresponds to the long arc detected in the $J-H$ magnitude image. This dark lane is due to an optically thick disk and the long arc appears due to this disk, where scattering occurs several times, resulting in low polarization.
The bottom panels in Fig. 1 show the degree of polarization images. The polarization vector lines are centro-symmetrically aligned in the outer part of the nebulosity. The degree of polarization there reaches $P_{J} \sim 30 \%, P_{H} \sim 42 \%$, and $P_{K} \sim 55 \%$. A polarization disk lies across the central star at a position angle of $\sim-55^{\circ}$. This is consistent with previous results of imaging polarimetry (Gledhill \& Scarrott 1989; Weintraub et al. 1995; Lucas et al. 2004). The polarization disk shows a butterfly shape with low polarizations of $P_{J}=1.6 \%, P_{H}=2.9 \%$, and $P_{K}=1.0 \%$ applying a $0 . ' 3$ diameter aperture. These values are lower than the results of $P_{H, K}=3-4 \%$ using the UKIRT and $P_{K}=6.8 \%$ using the Subaru presented by Lucas et al. (2004). The higher polarizations in their results are probably due to larger PSF sizes ( $0 . ' 4$ for both the UKIRT and Subaru data). Namely, scattered polarized fluxes around the central star contaminate the polarization measurement more at a larger PSF size. Therefore, our higher resolution data $\left(0,{ }^{\prime} 1-0 . ' 2\right)$ would show more realistic polarization values. The extension of the polarization disk slightly depends on the wavelength. The angular size is a $0 . ' 9$ in the direction perpendicular to the disk plane and $3{ }^{\prime \prime} .9$, 2 '. 5, and 1".7 parallel to the disk plane in the $J, H$, and $K$ bands, respectively, if the region of the polarization lower than $5 \%$ is measured. The threshold polarization to determine the polarization disk is not defined. Instead, a polarization disk is regarded as the lower polarization region, which resulted in multiple scattering and the regime is roughly a region of an optical depth higher than 1.0. In many observational results, the extension of a polarization disk is larger than the estimated dust disk size. In fact, previous millimeter and centimeter radio interferometric images estimated a $100 \mathrm{AU}$ radius or an even smaller size of $65 \mathrm{AU}$ for HL Tau's (Keplerian accretion) disk (e.g. Mundy et al. 1996; Wilner et al. 1996; Greaves et al. 2008, and references therein), resulting in an expected extention (diameter) of 0.9 to $11^{\prime \prime} 4$ at a distance of $140 \mathrm{pc}$ (Kenyon et al. 1994).

\section{Discussion}

\subsection{Grain size}

We estimate the grain sizes of HL Tau from our data. The high near-infrared polarization in the nebulosity suggests submicron-sized grains. On the other hand, previous observations have shown evidence of large grains from a low dust-opacity index derived from a millimeter flux excess (Beckwith \& Sargent 1991; Rodmann et al. 2006). Thus, we examine them separately.

We first investigated the grains in the nebulosity by means of one-dimensional single scattering modeling (Murakawa et al. 2008 b,c). We assumed the following model geometry parameters: an inner radius of $0.05 \mathrm{AU}$, a luminosity of $\sim 10 L_{\odot}$ (e.g. Robitaille et al. 2007), an outer radius of 1300 AU (Hayashi et al. 1993), a radial dust density distribution of $\rho(R) \propto R^{-1.5}$, and an optical depth of 1 at $V$ band. For dust grains, we assumed the DL-chemistry (Draine \& Lee 1984) and a powerlaw size distribution of $n(a) \propto a^{-3.5}$ with a size range of $0.005 \leq a \leq a_{\max }$. Because the lower limit of the grain size is insensitive in our modeling, we determined only the maximum value $a_{\max }$. We examined $a_{\max }$ values from 0.2 to $1.0 \mu \mathrm{m}$ with $0.1 \mu \mathrm{m}$ increments and calculated the degree of polarization. We compared these model results with our observations and found that $a_{\max }=0.4 \mu \mathrm{m}$ is a reasonable solution. Although the accuracy of the estimation from our polarization fit is actually quite good (about $\pm 0.1 \mu \mathrm{m}$ for submicron sizes), one should consider that: (1) the real chemical composition of dust in HL Tau nebula could be somewhat different from the DL-chemistry. For 

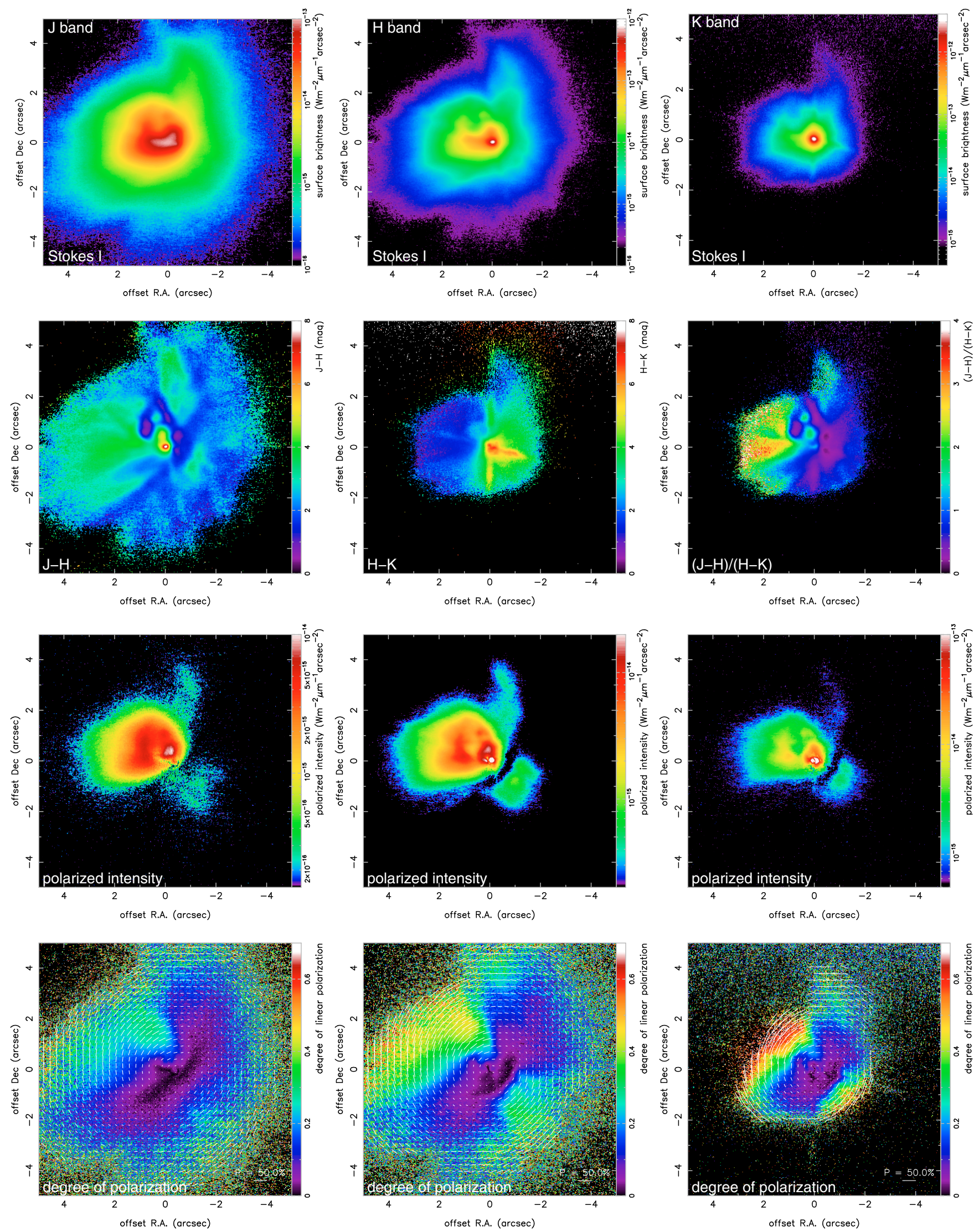

Fig. 1. $J, H$, and $K$ band polarimetric data of HL Tau obtained using CIAO on the Subaru telescope. The field of view is $10^{\prime \prime} \times 10^{\prime \prime}$. The top is north and left to the east. The top panels are the total intensity (Stokes $I$ ) images. The second row presents the $J-H$ and $H-K$ magnitude images and the $(J-H) /(H-K)$ image. The third row and the bottom panels show the polarized intensity images and the degree of polarization images with polarization vector lines, respectively. The X-shaped feature seen in the $H-K$ magnitude image is the diffraction pattern of the supporter of the telescope secondary mirror. 
instance, a water ice mantle is omitted in our modeling; (2) our single scattering modeling fits the polarization in a region where a centro-symmetric polarization vector pattern is seen. However, this sampling region does not have a large enough separation from the central star due to the data quality. Thus, the data could be diluted somewhat by multiple scattering components.

We estimated the grain size in the region close to the central star using the color excess ratio presented in Sect. 2.2. This value can be determined with the extinction cross section $C_{\lambda}$ as $E_{J-H} / E_{H-K}=\left(C_{J}-C_{H}\right) /\left(C_{H}-C_{K}\right)$. We calculated this for the above grain model with $a_{\max }$ values of 0.2 to $10.0 \mu \mathrm{m}$. Because the extinction efficient $Q_{\text {ext }}$ converges to 1 for size parameter $x(=2 \pi a / \lambda) \gg 1$, the $E_{J-H} / E_{H-K}$ also becomes a constant value, which is found to be 1.1 in our calculation and is consistent with our observational result. The corresponding grain size for this result is $a_{\max } \gtrsim 0.7 \mu \mathrm{m}$. Lucas et al. (2004) applied a single grain model of spheroidal shape in the entire nebula and found sizes slightly larger than $1 \mu \mathrm{m}$ and a grain axis ratio of 1.015 based on their polarization data, which shows higher values than ours. If our high-angular-resolution data and multiple dust models are applied, more spherical shapes and/or larger sizes are expected.

\subsection{Infrared polarization}

Previous infrared polarization of YSOs has derived several important results. (1) Objects are divided into two groups: whether the polarization orientation $\theta$ is parallel to the interstellar polarization or not. In the latter group, $\theta$ is often perpendicular to the $\mathrm{CO}$ outflow, suggesting that the polarization is governed by scattered light from the nebulosity. HL Tau belongs to this group (Hodapp 1984; Bastien 1987). (2) The polarization $P_{\lambda}$ as a function of wavelength follows (modified) Serkowski's law as well as field stars do (Serkowski et al. 1975). (3) The polarization efficiency $P_{\lambda} / \tau_{\lambda}$ is proportional to $A_{V}^{-0.52}$ and varies from $\sim 1-10 \%$ for objects in the Taurus dark cloud complex (Whittet et al. 2008). In their samples, the behavior of $P_{\lambda} / \tau_{\lambda}$ is scattered at large $A_{V}(\geq 10 \mathrm{mag})$ and enhanced up to a factor of 6 compared to the field stars.

The main interests of these studies are grain alignment mechanisms and magnetic field interaction with star formation. We propose adding another point of the grain size in data which has spatial information. The above infrared polarimetry has been obtained applying large apertures, which acquire almost entire flux from the source. Thus, the results are affected by the polarized flux from the nebula, where small grains (i.e. sub-micron-size) are expected to exist, and show similar polarization properties to the interstellar polarization, i.e. a strong wavelength dependence. We compare some results of HL Tau and other object of high-resolution data. In HL Tau, the $P_{\lambda}$ is less than $3 \%$ at the central star using a 0.' 3 diameter aperture and does not follow Serkowski's law. Because of this result, the polarization efficiency is also as low as $0.23,0.55$, and 0.27 in the $J H K$ bands, respectively, applying the optical depth estimated by Lucas et al. (2004). These results are explained by the presence of large grains in the disk. Murakawa et al. (2008c) presented the JHKband imaging polarimetry of the Herbig Be star R Mon. Their results also show low polarizations of $P_{J}=7 \%, P_{H}=2 \%$, and $P_{K}=1 \%$. Radio observation has shown evidence of large grains from a millimeter flux excess (Fuente et al. 2006). Thus, interpreting infrared polarization of this object is similar to doing it for HL Tau. On the other hand, other objects such as AFGL 2519 (Minchin et al. 1991), AFGL 437 (Meakin et al. 2005), and CRL 2136 (Murakawa et al. 2008b), which are probably higher mass than R Mon, show very different results. Although, in these data, the polarization disk is spatially resolved and the central star feature is seen, the polarization is still high $(P \gtrsim 20 \%)$ and the polarization vectors towards the central star are systematically aligned. For CRL 2136, Murakawa et al. (2008b) estimated the grain size $\left(a_{\max }\right)$ to be $0.45 \mu \mathrm{m}$ from their radiative transfer modeling. They also discussed the possible mechanisms of polarization vector alignment and concluded that the high polarization is probably produced by dichroic absorption by small non-spherical grains.

The aforementioned results suggest that many protostars of low- to intermediate-counterparts show low infrared polarization in the polarization disk and evidence of large grains, and the opposed results are seen in many massive stars. High-resolution near-infrared imaging polarimetry is a potential technique for investigating grain growth in the disk. Further studies of more samples and detailed modeling will provide us with new insights into the disk environment to determine grain growth and the magnetic field interaction in the disk and envelope.

Acknowledgements. Based on data collected at the Subaru telescope, which is operated by the National Astronomical Observatory of Japan.

\section{References}

Adams, F., Lada, C. J., \& Shu, F. 1987, ApJ, 312, 788

Bastien, P. 1987, ApJ, 317, 231

Bastien, P., \& Ménard, F. 1988, ApJ, 326, 334

Beckwith, S. V. W., \& Sargent, A. I. 1991, ApJ, 381, 250

Beckwith, S. V. W., \& Birk, C. C. 1995, ApJ, 449, L59

Bertout, C., Basri, G., \& Bouvier, J. 1998, ApJ, 330, 350

Calvet, N., Hartmann, L., Kenyon, S. J., \& Whitney, B. A. 1994, ApJ, 434, 330

Close, L. M., Roddier, F., Northcott, M. J., Roddier, C., \& Graves, J. E. 1997, ApJ, 478, 766

Draine, B. T., \& Lee, H. M. 1984, ApJ, 285, 89

Fischer, O., Henning, Th., \& Yorke, H. W. 1994, A\&A, 308, 863

Fuente, A., Alonso-Albi, T., Bachiller, et al. 2006, ApJ, 649, L119

Gledhill, T. M., \& Scarrott, S. M. 1989, MNRAS, 236, 139

Greaves, J., Richards, A. M. S., Rice, W. K. M., \& Muxlow, T. W. B. 2008, MNRAS, in press

Hayashi, M., Ohashi, N., \& Miyama, S. 1993, ApJ, 418, L71

Hodapp, K.-H. 1984, A\&A, 141, 255

Kenyon, S. J., \& Hartmann, L. 1987, ApJ, 323, 714

Kenyon, S. J., Whitney, B. A., Gomez, M., \& Hartomann, L. 1993, ApJ, 414, 773

Kenyon, S. J., Dobrzycka, D., \& Hartmann, L. 1994, AJ, 108, 1872

Lucas, P. W., Fukagawa, M., Tamura, M., et al. 2004, MNRAS, 352, 1347

Meakin, C. A., Hines, D. C., \& Thompson, R. I. 2005, ApJ, 634, 1146

Men'shchikov, A. B., Henning, T., \& Fischer, O. 1999, ApJ, 519, 257

Minchin, N. R., Hough, J. H., McCall, A., et al. 1991, MNRAS, 251, 508

Mundt, R., \& Fried, J. W. 1983, ApJ, 274, L83

Mundy, L. G., Looney, L. W., Erickson, W., et al. 1996, ApJ, 464, L169

Murakawa, K., Suto, H., Tamura, M., et al. 2004, PASJ, 56, 509

Murakawa, K., Suto, H., Oya, S., et al. 2005, A\&A, 436, 601

Murakawa, K., Ohnaka, K., Driebe, T., et al. 2008a, A\&A, 489, 195

Murakawa, K., Preibisch, T., Kraus, S., \& Weigelt, G. 2008b, A\&A, 490, 673

Murakawa, K., Preibisch, T., Kraus, S., et al. 2008c, A\&A, 488, L75

Perrin, M. D., Graham, J. R., Kalas, P., et al. 2004, Science, 3003, 1345

Pyo, T.-S., Hayashi, M., Kobayashi, N., et al. 2006, ApJ, 649, 836

Ray, T. P., Mundt, R., Dyso, J. E., Sam, A. E. G., \& Raga, A. C. 1996, ApJ, 468, L103

Robitaille, T. P., Whitney, B. A., Indebetouw, R., \& Wood, K. 2007, ApJS, 169, 328

Rodmann, J., Henning, Th., Chandler, C. J., Mundy, L. G., \& Wilner, D. J. 2006, A\&A, 446, 211

Serkowski, K., Mathewson, D. S., \& Ford, V. L. 1975, ApJ, 196, 261

Stapelfeldt, K. R., Burrows, C. J., Kris, J. E., et al. 1995, ApJ, 449, 888

Weintraub, D. A., Kastner, J. H., \& Whitney, B. A. 1995, ApJ, 452, L141

Whitney, B. A., \& Hartmann, L. 1993, ApJ, 402, 605

Whitney, B. A., Kenyon, S. J., \& Gomez, M. 1997, ApJ, 485, 703

Whittet, D. C. B., Martin, P. G., Hough, J. H., et al. 1992, ApJ, 386, 562

Whittet, D. C. B., Shenoy, S. S., Bergin, E. A., et al. 2007, ApJ, 655, 332

Whittet, D. C. B., Shenoy, Hough, J. H., Lazarian, A., \& Hoang, T. 2008, ApJ, 674,304

Wilner, D. J., Ho, P. T. P., \& Rodríguez, L. F. 1996, ApJ, 470, L117 